\title{
Reference gene selections for real time quantitative PCR analysis of gene expression in different oat tissues and under salt stress
}

\author{
Z.L. DUAN ${ }^{1}$, W.H. HAN ${ }^{2}$, L. YAN ${ }^{2}$, and B. $\mathrm{WU}^{2 *}$ \\ Yan'an Institute of Agricultural Sciences, Yan'an 716000, P.R. China ${ }^{1}$ \\ Institute of Crop Sciences, Chinese Academy of Agricultural Sciences, Beijing 100081, P.R. China ${ }^{2}$
}

\begin{abstract}
Appropriate choice of reference genes for data normalization is of critical importance for accurate real time reverse transcription quantitative PCR (RT-qPCR) analysis of gene expression. Oat is an agriculturally important crop cultivated widely around the world for grain or forage, and appropriate reference genes for reliable gene expression analysis remain to be identified. In this study, we selected nine candidate reference genes based on available oat RNA-seq data. We then conducted a systematic evaluation of the relative expression stability of these genes in different tissues and under salt stress using statistical algorithms, geNorm, NormFinder, and BestKeeper. Our findings reveal that the highest-ranked reference genes for accurate data normalization should be selected according to specific sample subsets. For different tissues, the combination of two reference genes [elongation factor 1-alpha $(E F 1 \alpha)$ and serine/threonine protein phosphatase 2] was sufficient for accurate normalization. For salt stress treatment, the combination of two reference genes (EF1 $\alpha$ and TATAbinding protein) was sufficient for accurate normalization. Moreover, the commonly used reference genes, actin and glyceraldehyde 3-phosphate dehydrogenase, were least suitable for data normalization of oat samples. Expression of a salt stress-inducible transcription factor Avena sativa WRKY2 was investigated to validate the reference genes identified in this study. This is the first systematic study of reference gene selection in cultivated oat and provides guidelines to obtain more accurate RT-qPCR results in this species.
\end{abstract}

Additional key words: Avena sativa, BestKeeper, geNorm, NormFinder, RT-qPCR.

\section{Introduction}

Transcriptomes and gene expression analyses have contributed greatly to our understanding of the complex regulatory networks underlying developmental and cellular processes. The variety of techniques available for analysis of gene expression includes Northern blotting, RNase protection, in situ hybridization, and reverse transcription quantitative PCR (RT-qPCR) (Bustin 2000). Due to its high sensitivity and accuracy, RT-qPCR is one of the most widely used techniques for analyzing gene expression (Sgamma et al. 2016). However, the accuracy of RT-qPCR is influenced by various parameters, such as the differences in quality and quantity of the initial RNA sample, as well as the efficiency of the reverse transcription and amplification reactions (Pfaffl et al. 2004, Udvardi et al. 2008). These biases in RT-qPCR analysis are commonly minimized by normalization of the target gene raw data against that of one or more internal controls.
Ideally, such control genes should be constitutively and stably expressed across different tissues, developmental stages, and environmental conditions (Vandesompele et al. 2002). Reference genes, which encode protein products that are involved in basic cellular processes such as cell structure maintenance or primary metabolism, fulfill these requirements and are widely used as internal control for RT-qPCR analyses. Common examples of reference genes include actin, tubulin, ubiquitin, and glyceraldehyde3-phosphate dehydrogenase. However, accumulating evidences indicate that reference gene expression is influenced by experimental conditions (Suzuki et al. 2000, Kozera and Rapacz 2013), highlighting the importance of careful selection of validated reference genes (Guénin et al. 2009, Hruz et al. 2011).

Cultivated oat (Avena sativa L.) is a grass species grown as a grain or forage crop predominantly in temperate short-season regions (Loskutov and Rines 2011). In recent decades, the discovery of the nutrient-rich and cholesterol-

Submitted 14 July 2020, last revision 25 September 2020, accepted 15 October 2020.

Abbreviations: ACT - actin; CYP - cyclophilin; EF1 $\alpha$ - elongation factor 1-alpha; GAPDH - glyceraldehyde-3-phosphate dehydrogenase; PP2A - serine/threonine protein phosphatase 2A; RT-qPCR - reverse transcription quantitative PCR; TBP - TATA-binding protein; TUA - tubulin alpha chain; TUB - tubulin beta chain; UBQ - ubiquitin.

Acknowledgements: This work was supported by the Chinese Agricultural Research System (grant No. CARS-07-A-1) and the National Natural Science Foundation of China (grant No. 30800699).

* Corresponding author; e-mail: wubin03@caas.cn 
lowering properties has led to wider appreciation of oat for health-promoting compounds, such as beta-glucan, avenanthramides, vitamin E, and phytic acid (Xiao et al. 2015, Smulders et al. 2017). Moreover, oat is a dominant crop in some marginal areas of northern and western China that are affected by salinity and aridity, and is expected to become a pioneer crop for desertification control. However, understanding molecular mechanism of abiotic stress tolerance requires analyzing the gene expression profile under stress. The reliability of the data generated in such studies is critically dependent on the identification of suitable reference genes. Such internal controls have been identified and validated for a variety of plant species (Paolacci et al. 2009, Manoli et al. 2012, Saha et al. 2014, Acevedo et al. 2018). However, to our knowledge, no systematic evaluation of appropriate reference genes in cultivated oat has yet been reported. To solve this problem, we conducted a systematic investigation of 9 candidate genes for accurate RT-qPCR data normalization in cultivated oat. The candidates evaluated in this study included commonly used reference genes and potential genes selected from previous RNA-seq data (Wu et al. 2017). With the aim to validate our findings, the expression of a salt stress-inducible transcription factor (Avena sativa WRKY2) was analyzed following normalization against the different candidate reference genes.

\section{Materials and methods}

Plants and treatments: Oat (Avena sativa L.) cv. BY-1 seeds were sterilized with $1 \%(\mathrm{~m} / \mathrm{v})$ sodium hypochlorite, rinsed with distilled water, and sown in plastic pots filled with standard soil. The seedlings were cultivated in a greenhouse under a photon flux density of $240 \mu \mathrm{mol} \mathrm{m} \mathrm{m}^{-2} \mathrm{~s}^{-1}$, a 18 -h photoperiod, day/night temperatures of $25 / 16^{\circ} \mathrm{C}$, and a relative humidity of $60 \%$. For salt stress experiments, plants at the two-leaf stage were watered with $2 \%(\mathrm{~m} / \mathrm{v})$ $\mathrm{NaCl}$ solution. Seedlings were harvested after plants exposure to $\mathrm{NaCl}$ for $0,0.5,1,2,4,8,12$, and $24 \mathrm{~h}$. For the evaluation of different tissues, roots, stems, leaves, and spikelet were collected. All samples were immediately frozen in liquid nitrogen and stored at $-80{ }^{\circ} \mathrm{C}$ prior to further analyses.

Isolation of RNA and cDNA synthesis: Total RNA was extracted using Trizol reagent (Invitrogen, Carlsbad, USA). RNA purity and concentration were determined using NanoPhotometer P360 (Implen, Munich, Germany), and integrity was assessed by $1 \%(\mathrm{~m} / \mathrm{v})$ agarose gel electrophoresis. First-strand cDNA was synthesized from total RNA using GoScript ${ }^{\mathrm{TM}}$ reverse transcription kit (Promega, Madison, WI, USA) according to the manufacturer's instructions. All cDNA samples were diluted to $100 \mathrm{ng} \mathrm{mm}^{-3}$ for RT-qPCR.

Primer design for PCR: Nine candidate reference genes were selected based on published studies and previously reported RNA-seq data (Wu et al. 2017). These genes were cloned and sequenced to obtain accurate sequence information and specific primers were designed using Primer Express software v. 3.0.1 and Primer3 (Untergasser et al.2012); their characteristics are listed in Table 1 Suppl. Before starting RT-qPCR, PCRs were performed on oat cDNA with all designed primers. In order to verify the sequences of amplification products, PCR products were purified and cloned for sequencing. Sequences of amplification products were compared with original gene sequences. Only primers which amplified sequences and had $100 \%$ identities with corresponding gene sequences were selected for further RT-qPCR assay.

Real time quantitative PCR analysis was performed on the 7500 Real Time PCR System (Applied Biosystems, Foster City, CA, USA). The reaction mixtures consisted of $10 \mathrm{~mm}^{3}$ of PowerUp ${ }^{\mathrm{TM}}$ SYBR ${ }^{\mathrm{TM}}$ Green Master Mix (Thermo Fisher Scientific, Austin, TX), $400 \mathrm{nM}$ of each primer, and $100 \mathrm{ng}$ of cDNA. The reaction conditions were as follows: $50{ }^{\circ} \mathrm{C}$ for $2 \mathrm{~min}, 95^{\circ} \mathrm{C}$ for $10 \mathrm{~min}$, followed by 40 cycles of $95{ }^{\circ} \mathrm{C}$ for $15 \mathrm{~s}$ and $60{ }^{\circ} \mathrm{C}$ for $1 \mathrm{~min}$. Immediately after amplification, the specificity of the reactions was confirmed by melting curve analysis. All real-time PCR reactions were repeated at least three times. Amplification efficiency (E) was calculated for each primer pair according to the following formula: $\mathrm{E}[\%]=\left[10^{-(1 / \text { slope })}-1\right] \times 100$ (Niu et al. 2015). The slope was determined as the regression line between the quantification cycle $(\mathrm{Cq})$ values and the logarithm values of different cDNA concentrations (Moraes et al. 2015).

Data analysis: Stability of the candidate genes was assessed using geNorm, NormFinder, and BestKeeper. For geNorm and NormFinder, the raw $\mathrm{Cq}$ values were transformed into relative quantities (Q) according to the formula: $\mathrm{Q}=\mathrm{E}^{-\Delta \mathrm{Cq}}(\Delta \mathrm{Cq}=$ the corresponding $\mathrm{Cq}$ - the minimum Cq) (Niu et al. 2015). The raw $\mathrm{Cq}$ values were directly analyzed using the BestKeeper program.

The average expression stability (M values) of candidate genes was evaluated by geNorm, with a threshold of $M>1.5$ set for elimination. To select the optimal number of reference genes for normalization, pairwise variation $\left(\mathrm{V}_{\mathrm{n}} / \mathrm{V}_{\mathrm{n}+1}\right)$ was also calculated by geNorm (Vandesompele et al. 2002). The stability of candidate genes was evaluated by NormFinder (Andersen et al. 2004). Gene expression stability was also evaluated using BestKeeper by calculating the coefficient of variation $(\mathrm{CV})$ and standard deviation (SD) of the Cq values (Pfaffl et al. 2004).

Assessment of normalization: To validate the effectiveness of the selected reference genes, we used AsWRKY2 (accession number: MH260259) as a target gene and compared its relative expression when normalized against different reference genes. The RTqPCR procedure was conducted as described above using the following primers for amplification of AsWRKY2: GGGTTGGGATTTCCCTTGAC (forward primer) and CATACTCGACGACGGCTTCA (reverse primer). AsWRKY2 expressions were analyzed using the $2^{-\Delta \Delta \mathrm{Cq}}$ method (Livak et al. 2001). Means of different groups were compared and analyzed using Student's $t$-test and 
a priori alpha error $P$ of less than 0.05 was considered to indicate the statistical significance.

\section{Results}

Nine candidate genes [actin (ACT), glyceraldehyde 3-phosphate dehydrogenase (GAPDH), ubiquitin (UBQ), elongation factor 1-alpha (EF1 $\alpha)$, tubulin alpha chain (TUA), tubulin beta chain (TUB), cyclophilin (CYP), serinel threonine protein phosphatase $2 A(P P 2 A)$, and TATAbinding protein $(T B P)]$ were selected and corresponding PCR primers were designed. Gene accession numbers, primer sequences, and other amplicon parameters are listed in Table 1 Suppl. Amplification specificity was confirmed by the presence of a single peak (Fig. 1 Suppl.) in the melting curve for each PCR product, demonstrating the absence of primer dimers and other products resulting from non-specific amplification. The amplification efficiency for each primer pair exceeded $90 \%$, ranging between $91 \%(U B Q$ and $E F 1 \alpha)$ and $115 \%(A C T)$, with regression coefficients $\left(R^{2}\right)$ varying from $0.987(U B Q)$ to 0.9993 (TBP) (Table 1 Suppl.).

Expressions displayed as RT-qPCR $\mathrm{Cq}$ values for each candidate gene are shown in Fig. 1. Among all the tested samples, there were no significant differences in the $\mathrm{Cq}$ values for candidate genes, with an average value of 25.31 and values ranging from $19.72(C Y P)$ to 30.02 (TBP). CYP had the lowest mean Cq value (21.62), followed by TUA (23.92), which indicated these genes as more expressed genes across the nine candidate reference genes analyzed. In contrast, the highest median $\mathrm{Cq}$ value (28.29) was attributed to TBP gene, indicating its low expression across the tested samples.

The stability values for all reference genes calculated by geNorm, NormFinder, and BestKeeper are shown in Table 1. The geNorm program recommends using $M$ values below the threshold of 1.5 to identify stably expressed

Table 1. The expression stability ranking of nine candidate genes evaluated by three algorithms. Candidate reference gene expression stability ranked by geNorm, NormFinder, and BestKeeper in salt stressed groups and different tissue samples of oat. Candidate genes are listed from top to bottom in order of increasing expression stability. The comprehensive ranking was based on calculation of the geometric mean of geNorm, NormFinder, and BestKeeper results. M- average expression stability, SV - stability value, CV - coefficient of variance expressed as a percentage of the cycle threshold $(\mathrm{Ct})$ level, $\mathrm{SD}$ - standard deviation of the Ct; ACT - actin, GAPDH glyceraldehyde 3-phosphate dehydrogenase, UBQ - ubiquitin, EF1 $\alpha$ - elongation factor 1-alpha, TUA - tubulin alpha chain, TUB tubulin beta chain, CYP - cyclophilin, PP2A - serine/threonine protein phosphatase $2 A$, TBP - TATA-binding protein.

\begin{tabular}{|c|c|c|c|c|c|c|c|c|c|}
\hline \multirow[t]{2}{*}{ Experiment } & \multirow[t]{2}{*}{ Rank } & \multicolumn{2}{|l|}{ geNorm } & \multicolumn{2}{|c|}{ NormFinder } & \multicolumn{3}{|c|}{ BestKeeper } & \multirow[t]{2}{*}{ Comprehensive ranking } \\
\hline & & gene & M & gene & SV & gene & $\mathrm{CV}$ & SD & \\
\hline \multirow[t]{10}{*}{ Salt stressed samples } & 1 & $E F 1 \alpha$ & 0.209 & $P P 2 A$ & 0.270 & $T B P$ & 1.52 & 0.42 & $E F 1 \alpha$ \\
\hline & 2 & $T B P$ & 0.209 & $T U B$ & 0.270 & $E F 1 \alpha$ & 2.41 & 0.60 & $T B P$ \\
\hline & 3 & $U B Q$ & 0.386 & $E F 1 \alpha$ & 0.407 & $P P 2 A$ & 3.13 & 0.79 & $P P 2 A$ \\
\hline & 4 & $P P 2 A$ & 0.470 & $U B Q$ & 0.434 & $U B Q$ & 3.22 & 0.84 & $U B Q$ \\
\hline & 5 & $C Y P$ & 0.615 & $T B P$ & 0.560 & $C Y P$ & 3.39 & 0.69 & $T U B$ \\
\hline & 6 & $T U B$ & 0.796 & $G A P D H$ & 0.621 & $T U B$ & 3.89 & 0.93 & $C Y P$ \\
\hline & 7 & $G A P D H$ & 0.977 & $T U A$ & 1.001 & $G A P D H$ & 6.08 & 1.41 & $G A P D H$ \\
\hline & 8 & $T U A$ & 1.189 & $C Y P$ & 1.147 & $T U A$ & 9.18 & 2.09 & $T U A$ \\
\hline & 9 & $A C T$ & 1.571 & $A C T$ & 2.117 & $A C T$ & 11.09 & 2.76 & $A C T$ \\
\hline & 1 & $E F 1 \alpha$ & 0.242 & $P P 2 A$ & 0.220 & $T U B$ & 2.65 & 0.66 & $E F 1 \alpha$ \\
\hline \multirow[t]{10}{*}{ Different tissue samples } & 2 & $T B P$ & 0.242 & $T U B$ & 0.349 & $P P 2 A$ & 3.08 & 0.85 & $P P 2 A$ \\
\hline & 3 & $P P 2 A$ & 0.390 & $E F 1 \alpha$ & 0.530 & $E F 1 \alpha$ & 3.30 & 0.88 & $T U B$ \\
\hline & 4 & $U B Q$ & 0.507 & $T B P$ & 0.631 & $T B P$ & 3.40 & 0.99 & $T B P$ \\
\hline & 5 & $T U B$ & 0.758 & $U B Q$ & 0.817 & $U B Q$ & 5.01 & 1.40 & $U B Q$ \\
\hline & 6 & $C Y P$ & 0.858 & $C Y P$ & 1.008 & $T U A$ & 5.44 & 1.37 & $C Y P$ \\
\hline & 7 & $A C T$ & 1.195 & $A C T$ & 1.136 & $G A P D H$ & 6.34 & 1.64 & $T U A$ \\
\hline & 8 & $T U A$ & 1.455 & $T U A$ & 1.472 & $C Y P$ & 6.38 & 1.46 & $A C T$ \\
\hline & 9 & $G A P D H$ & 1.643 & $G A P D H$ & 1.480 & $A C T$ & 4.49 & 1.21 & $G A P D H$ \\
\hline & 1 & $E F 1 \alpha$ & 0.378 & $P P 2 A$ & 0.294 & $T B P$ & 3.42 & 0.97 & $E F 1 \alpha$ \\
\hline & 2 & $T B P$ & 0.378 & $T U B$ & 0.399 & $T U B$ & 3.91 & 0.96 & $P P 2 A$ \\
\hline \multirow[t]{7}{*}{ All samples } & 3 & $U B Q$ & 0.457 & $E F 1 \alpha$ & 0.433 & $P P 2 A$ & 4.55 & 1.20 & $T B P$ \\
\hline & 4 & $P P 2 A$ & 0.578 & $U B Q$ & 0.615 & $E F 1 \alpha$ & 4.68 & 1.21 & $T U B$ \\
\hline & 5 & $T U B$ & 0.779 & $T B P$ & 0.617 & $U B Q$ & 4.86 & 1.32 & $U B Q$ \\
\hline & 6 & $C Y P$ & 0.900 & $C Y P$ & 1.042 & $G A P D H$ & 6.52 & 1.60 & $C Y P$ \\
\hline & 7 & $G A P D H$ & 1.182 & $G A P D H$ & 1.092 & $C Y P$ & 6.99 & 1.51 & $G A P D H$ \\
\hline & 8 & $T U A$ & 1.389 & $T U A$ & 1.166 & $T U A$ & 7.74 & 1.85 & $T U A$ \\
\hline & 9 & $A C T$ & 1.602 & $A C T$ & 1.576 & $A C T$ & 8.67 & 2.25 & $A C T$ \\
\hline
\end{tabular}




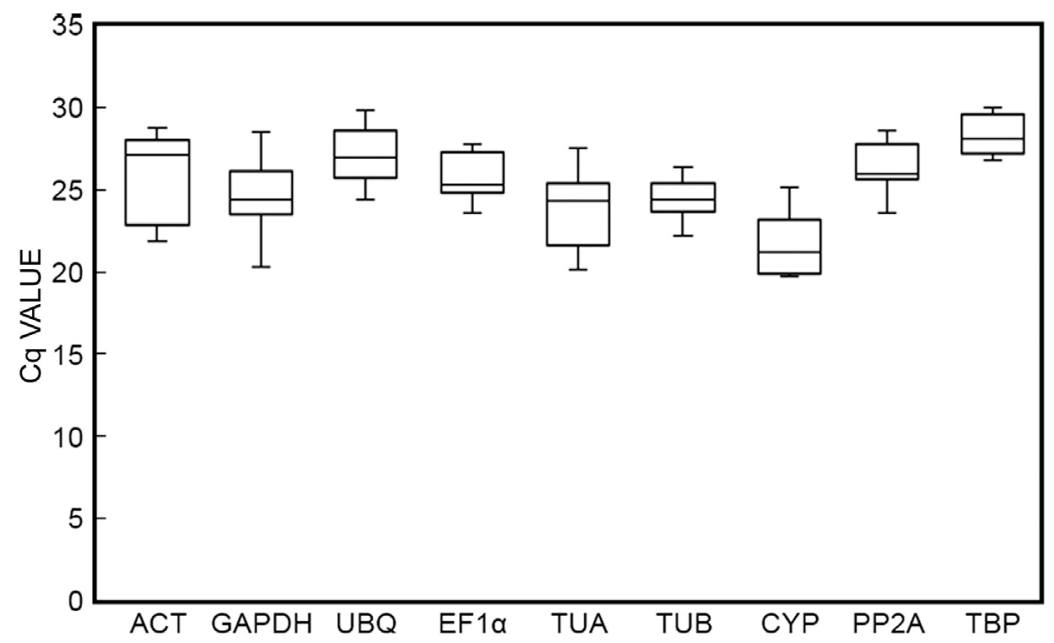

Fig. 1. Candidate reference gene expressions across all samples. Expressions displayed as reverse transcription PCR quantification cycle $(\mathrm{Cq})$ values for each candidate gene, maximum and minimum Cq values are indicated by whisker caps. Boxes indicate $25^{\text {th }}$ and $75^{\text {th }}$ percentiles with lines depicting medians.

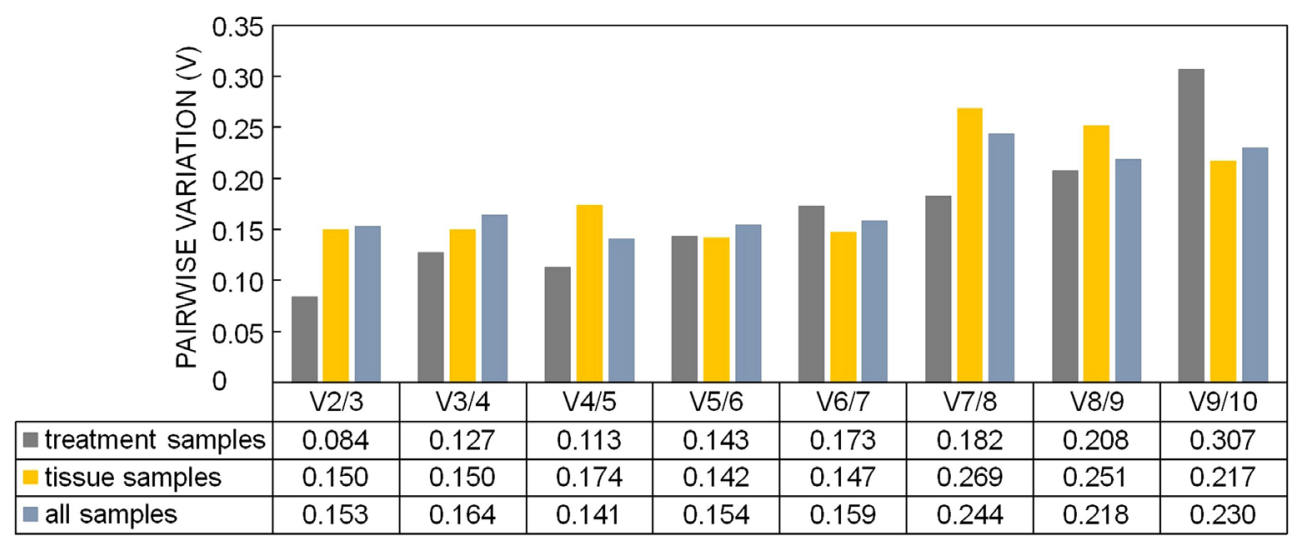

Fig. 2. Determination of the optimal number of reference genes required for effective normalization. The optimal number of reference genes required for effective normalization was determined by pairwise variation $\left(\mathrm{V}_{\mathrm{n} / \mathrm{n}+1}\right)$ analysis of the normalization factors (NFn and $\mathrm{NFn}+1$ ) with a recommended cutoff of 0.15 . The calculated pairwise variation values are tabulated.

reference genes. With the exception of GAPDH (1.643) and $A C T$ (1.602), all candidate genes analyzed in this study had $M$ values below 1.5, indicating the suitability of these genes as stable reference gene candidates. Under conditions of salt stress, EF $\alpha$ and $T B P$ were the most stable combination of all genes tested $(\mathrm{M}=0.209)$, followed by $U B Q$. Similar results were obtained for different tissue samples, although there were slight changes in the order of ranking. EF $1 \alpha$ and $T B P$ were the most stable two genes of all genes tested $(M=0.242)$ in the different tissue samples. For the combined datasets, EF1 $\alpha$ and TBP were also the most stable genes $(\mathrm{M}=0.378)$ (Table 1$)$.

Despite some variation in the stability ranking of the nine candidate genes, the results obtained using the NormFinder and geNorm algorithms exhibited marked similarities. For example, both methods identified $C Y P$, $G A P D H, T U A$, and $A C T$ as the four least stable genes and $E F 1 \alpha, U B Q, P P 2 A$, and $T B P$ as the four most stable. For the salt stress treated samples, NormFinder analysis ranked $P P 2 A$ and TUB as the first and second best genes for data normalization based on stability values, while
$A C T$ was the least stable. This pattern was repeated when all the tested samples were analyzed.

The results of the BestKeeper analysis shows that for the salt stress treated samples, the same three genes were identified by both the BestKeeper and geNorm programs, although their rank order was slightly altered. For the different tissue samples, TUB emerged as the most stably expressed gene. When evaluated across all tested samples, $T B P, T U B$, and $P P 2 A$ were in the top positions, whereas $A C T$ was ranked as least stable.

Despite the increased cost, the use of multiple reference genes offers the potential to generate much more accurate results. Therefore, it is important to identify the optimal number of reference genes for accurate normalization in qPCR assays. In this study, we selected the optimal reference genes based on the pairwise variation in two experimental sets of the samples determined using geNorm. The $\mathrm{N}$ values of $\mathrm{V}_{\mathrm{n} / \mathrm{n}+1}$ below 0.15 were considered to be the optimal number of control genes for reliable normalization (Vandesompele et al. 2002). Using this cutoff in stress treatments $\left(\mathrm{V}_{2 / 3}=0.084\right)$ and different tissue samples 


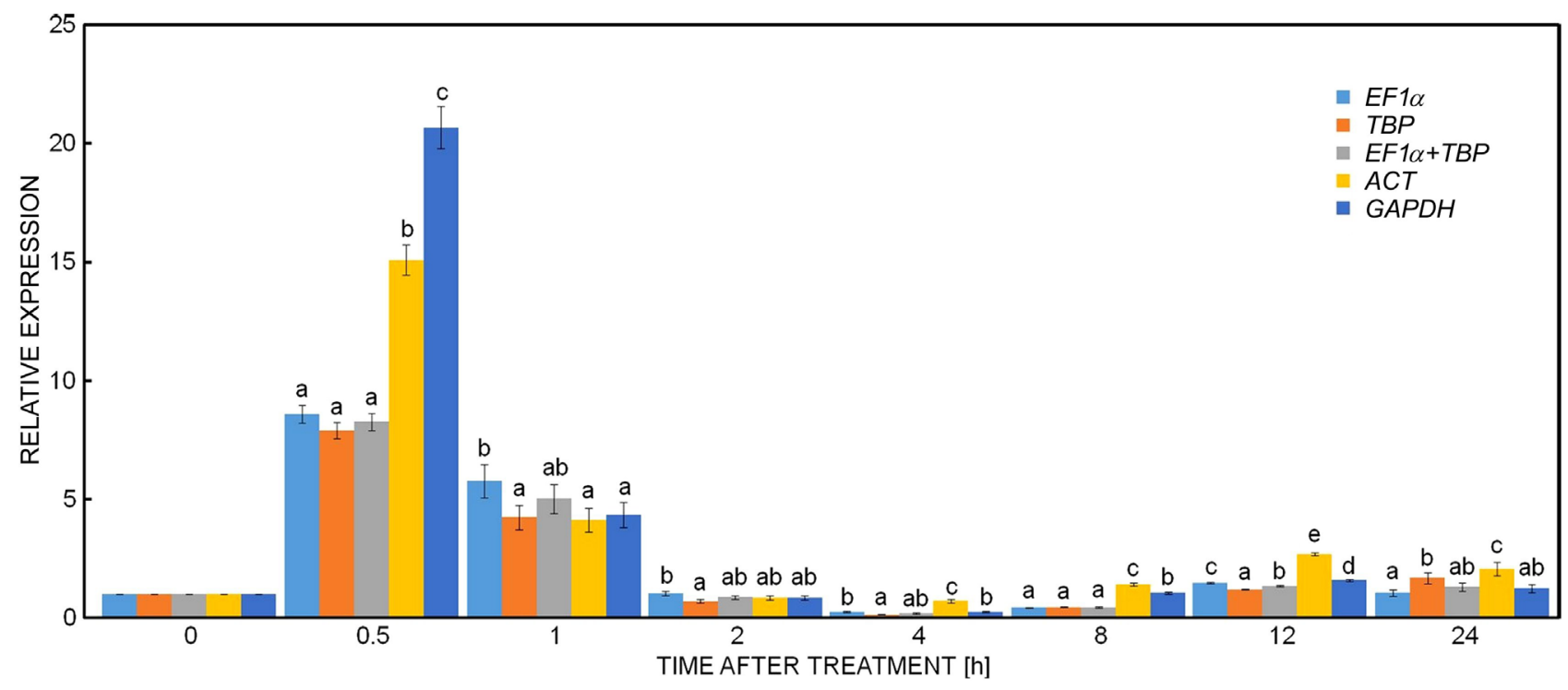

Fig. 3. Relative expression of a stress responsive Avena sativa WRKY2 gene in response to salt stress. Expressions of AsWRKY2 gene at seven different time-points following salt stress treatment. Data were normalized against the best selected genes, elongation factor 1-alpha and TATA-binding protein, both individually and in combination, and the least stable genes, actin and glyceraldehyde 3-phosphate dehydrogenase. Data represent mean fold changes in relative expressions compared with a control $\pm \operatorname{SDs}(n=3)$. Different letters denote statistically significant differences $(P<0.05)$.

$\left(\mathrm{V}_{2 / 3}=0.150\right)$ indicated that the combination of the two most stable genes was optimal for normalization of gene expression data. However, when all experimental samples were considered, the pairwise variation $\mathrm{V}_{2 / 3}$ exceeded $0.15(0.153)$, whereas $\mathrm{V}_{4 / 5}$ was 0.141 , indicating that four reference genes $(E F 1 \alpha+P P 2 A+T B P+T U B)$ were optimal for the normalization if data from different tissues will be considered in a salt stress experiment (Fig. 2).

To validate the reliability of the selected reference genes in cultivated oat, the relative expressions of a stress-inducible gene, AsWRKY2, were detected. RTqPCR data were normalized against the most highly ranked genes individually and then in combination. The commonly used reference genes, $A C T$ and $G A P D H$, which showed the least stability in this study, were also selected for normalization. Fig. 3 shows that the relative transcript abundance was overestimated in five of the eight stages of salinity stress $(0.5,4,8,12,24 \mathrm{~h})$. Moreover, after $8 \mathrm{~h}$, the AsWRKY2 expression profile normalized against $A C T$ differed obviously from expression profiles generated by normalization with other reference genes and the combination of EF1 $\alpha$ and TBP. Therefore, different AsWRKY2 expression profiles were obtained following normalization of the data according to the genes with the most and least stable expression selected by each program. These findings emphasized the importance of validating reference gene stability prior to its application in RT-qPCR assays.

\section{Discussion}

The influence of reference gene selection on the accuracy of RT-qPCR data was analyzed in many crops, such as rice, maize, and wheat (Remans et al. 2014). In cultivated oat, genes coding for $M D H$ (malate dehydrogenase) and $C D C 48$ (CDC48 ATPase) were chosen as internal control for identification of oat genes in response to Puccinia coronata infection, but no systematic evaluation of appropriate reference genes in cultivated oat under various conditions of development and salinity has been reported yet (Loarce et al. 2016). In this study, we conducted a systematic analysis of the expression stability of 9 candidate reference genes, including commonly used reference genes and some candidate genes selected from previous RNA-seq results (Wu et al. 2017). Using different statistical algorithms, we demonstrated differences in the relative quantification of target genes depending on the number and choice of reference genes (Fig. 3). Among the tested candidate genes, we identified the combination of $E F 1 \alpha+P P 2 A$ and $E F 1 \alpha+T B P$ as the most stable reference genes for RT-qPCR data normalization in gene expression studies of different tissue samples and salt stress, respectively.

In the present study, we demonstrated marked variability in the expression of the commonly used plant reference genes $A C T, G A P D H$, and $T U A$. This lack of expression stability suggests that these genes are unsuitable for use as reference genes in RT-qPCR studies of oat. For example, $A C T$ expression stability was shown to be highly variable across salinity treated oat sample sets. Furthermore, $A C T$ was ranked as the least stable gene in all the experimental sample sets by geNorm, NormFinder, and BestKeeper (Table 1). It can be speculated that this variability is caused by the function of actin, the product of $A C T$, in a variety of cellular processes in addition to its role as a major structural component of cellular microfilaments (Stürzenbaum and Kille 2001). Similarly, GAPDH was ranked as the least 
stable gene by geNorm and NormFinder across the given set of tissues, and is therefore not considered suitable for use as a reference gene in oat samples. These results emphasize the importance of reliable reference gene selection for normalization of RT-qPCR data and highlight the fact that this selection cannot be extrapolated across species.

In this study, we used three different statistical algorithms to minimize possible bias in the reference gene stability evaluation. In accordance with previous studies, the geNorm, NormFinder, and BestKeeper programs yielded slight differences in the ranking of the reference genes, which can be accounted for by differences in the statistical algorithms (Vandesompele et al. 2002). Since these statistical programs are complementary, we combined the results obtained into a comprehensive ranking by calculating as the geometric mean of multiple candidate genes, which currently represents the gold standard in such evaluations (Nolan et al. 2006).

Previous studies have shown that the accuracy of data normalization can be improved by using multiple reference genes (Vandesompele et al. 2002, Kozera and Rapacz 2013). Since the results of our analysis using the different statistical algorithms did not allow us to make a conclusive selection of a single reference gene appropriate, we calculated the corresponding RT-qPCR assay normalization factors. GeNorm analysis yielded $\mathrm{V}_{2 / 3} \mathrm{NF}$ values below 0.15 (Fig. 2), indicating that the combination of at least two reference genes is required for reliable normalization of RT-qPCR data for different tissue samples and salt-treated sample sets in oat. For salttreated samples, EF1 $\alpha+T B P$ were identified as the most appropriate combination by geNorm, while NormFinder included PP2A. However, BestKeeper analysis revealed much higher $\mathrm{CV}$ and SD values for $P P 2 A$ than $E F 1 \alpha+T B P$. For analysis of different tissue samples, the $E F 1 \alpha+P P 2 A$ combination was recommended.

The suitability of the selected reference genes was validated by comparing different reference genes both individually and in combination for RT-qPCR data normalization in the analysis of AsWRKY2 gene expression. The WRKY superfamily of transcription factors is involved in the regulation of various biological processes, especially stress responses (Jiang et al. 2017). In wheat, WRKY2 was found to be induced under salt treatment (Niu et al. 2012). Digital expression analyses of the oat transcriptome also confirmed that salt stress induced WRKY2 expression (Wu et al. 2017). Our study showed significant differences in AsWRKY2 gene expression data at some stages under salt stress following normalization against $E F 1 \alpha+T B P$ compared with those obtained using $A C T$ and $G A P D H$. However, similar results were obtained using EF $\alpha$ and TBP individually or in combination.

\section{References}

Acevedo, R.M., Avico, E.H., Ruiz, O.A., Sansberro, P.A.: Assessment of reference genes for real-time quantitative PCR normalization in Ilex paraguariensis leaves during drought. -
Biol. Plant. 62: 89-96, 2018.

Andersen, C.L., Jensen, J.L., Ørntoft, T.F.: Normalization of realtime quantitative reverse transcription-PCR data: a modelbased variance estimation approach to identify genes suited for normalization, applied to bladder and colon cancer data sets. - Cancer Res. 64: 5245-5250, 2004.

Bustin, S.A.: Absolute quantification of mRNA using real-time reverse transcription polymerase chain reaction assays. J. mol. Endocrinol. 25: 169-193, 2000.

Guénin, S., Mauriat, M., Pelloux, J., Van Wuytswinkel, O., Bellini, C., Gutierrez, L.: Normalization of qRT-PCR data: the necessity of adopting a systematic, experimental conditionsspecific, validation of references. - J. exp. Bot. 60: 487-493, 2009.

Hruz, T., Wyss, M., Docquier, M., Pfaffl, M.W., Masanetz, S., Borghi, L., Verbrugghe, P., Kalaydjieva, L., Bleuler, S., Laule, O., Descombes, P., Gruissem, W., Zimmermann, P.: RefGenes: identification of reliable and condition specific reference genes for RT-qPCR data normalization. - BMC Genomics 12: 156, 2011.

Jiang, J., Ma, S., Ye, N., Jiang, M., Cao, J., Zhang, J.: WRKY transcription factors in plant responses to stresses. - J. integr. Plant Biol. 59: 86-101, 2017.

Kozera, B., Rapacz, M.: Reference genes in real-time PCR. - J. appl. Genet. 54: 391-406, 2013.

Livak, K.J., Schmittgen, T.D.: Analysis of relative gene expression data using real-time quantitative PCR and the 2 $-\triangle \Delta \mathrm{CT}$ method. - Methods 25: 402-408, 2001.

Loarce, Y., Navas, E., Paniagua, C., Fominaya, A., Manjón, J.L., Ferrer, E.: Identification of genes in a partially resistant genotype of Avena sativa expressed in response to Puccinia coronata infection. - Front. Plant Sci. 7: 731, 2016.

Loskutov, I.G., Rines, H.W.: Avena. - In: Kole, C. (ed.): Wild Crop Relatives: Genomic and Breeding Resources. Pp.109183. Springer, Berlin 2011.

Manoli, A., Sturaro, A., Trevisan, S., Quaggiotti, S., Nonis, A.: Evaluation of candidate reference genes for qPCR in maize. J. Plant Physiol. 169: 807-815, 2012.

Moraes, G.P., Benitez, L.C., Do Amaral, M.N., Vighi, I.L., Auler, P.A., Da Maia, L.C., Bianchi, V.J., Braga, E.J.B.: Evaluation of reference genes for RT-qPCR studies in the leaves of rice seedlings under salt stress. - Genet. mol. Res. 14: 2384-2398, 2015.

Niu, C.F., Wei, W., Zhou, Q.Y., Tian, A.G., Hao, Y.J., Zhang, W.K., Ma, B., Lin, Q., Zhang, Z.B., Zhang, J.S., Chen, S.Y.: Wheat WRKY genes TaWRKY2 and TaWRKY19 regulate abiotic stress tolerance in transgenic Arabidopsis plants. Plant Cell Environ. 35: 1156-1170, 2012.

Niu, X.P., Qi, J.M., Zhang, G.Y., Xu, J.T., Tao, A.F., Fang, P.P., Su, J.G.: Selection of reliable reference genes for quantitative real-time PCR gene expression analysis in jute (Corchorus capsularis) under stress treatments. - Front. Plant Sci. 6: 848, 2015.

Nolan, T., Hands, R.E., Bustin, S.A.: Quantification of mRNA using real-time RT-PCR. - Nat. Protocols 1: 1559-1582, 2006.

Paolacci, A.R., Tanzarella, O.A., Porceddu, E., Ciaffi, M.: Identification and validation of reference genes for quantitative RT-PCR normalization in wheat. - BMC mol. Biol. 10: 11, 2009.

Pfaffl, M.W., Tichopad, A., Prgomet, C., Neuvians, T.P.: Determination of stable housekeeping genes, differentially regulated target genes and sample integrity: BestKeeperExcel-based tool using pair-wise correlations. - Biotechnol. Lett. 26: 509-515, 2004.

Remans, T., Keunen, E., Bex, G.J., Smeets, K., Vangronsveld, J., Cuypers, A.: Reliable gene expression analysis by reverse 
transcription-quantitative PCR: reporting and minimizing the uncertainty in data accuracy. - Plant Cell 26: 3829-3837, 2014. Saha, P., Blumwald, E.: Assessing reference genes for accurate transcript normalization using quantitative real-time PCR in pearl millet [Pennisetum glaucum (L.) R. Br]. - PLoS ONE 9: e106308, 2014.

Sgamma, T., Pape, J., Massiah, A., Jackson, S.: Selection of reference genes for diurnal and developmental time-course real-time PCR expression analyses in lettuce. - Plant Methods 12: 21, 2016.

Smulders, M.J.M., Van de Wiel, C.C.M., Van den Broeck, H.C., Van der Meer, I.M., Israel-Hoevelaken, T.P.M., Timmer, R.D., Braun, S., Gilissen, L.J.W.J.: Oats in healthy gluten-free and regular diets: a perspective. - Food Res. Int. 110: e0196334, 2017.

Stürzenbaum, S.R., Kille, P.: Control genes in quantitative molecular biological techniques: the variability of invariance. - Comp. Biochem. Physiol. B Biochem. mol. Biol. 130: 281289, 2001.

Suzuki, T., Higgins, P.J., Crawford, D.R.: Control selection for
RNA quantitation. - Biotechniques 29: 332-337, 2000.

Udvardi, M.K., Czechowski, T., Scheible, W.R.: Eleven golden rules of quantitative RT-PCR. - Plant Cell 20: 736-1737, 2008.

Untergasser, A., Cutcutache, I., Koressaar, T., Ye, J., Faircloth, B.C., Remm, M., Rozen, S.G.: Primer3-new capabilities and interfaces. - Nucl. Acids Res. 40: e115, 2012.

Vandesompele, J., De Preter, K., Pattyn, F., Poppe, B., Van Roy, N., De Paepe, A., Speleman, F.: Accurate normalization of real-time quantitative RT-PCR data by geometric averaging of multiple internal control genes. - Genome Biol. 3: research0034.1, 2002.

Wu, B., Hu, Y., Huo, P., Zhang, Q., Chen, X., Zhang, Z.: Transcriptome analysis of hexaploid hulless oat in response to salinity stress. - PLoS ONE 12: e0171451, 2017.

Xiao, Y., Rui, X., Xing, G., Wu, H., Li, W., Chen, X., Jiang, M., Dong, M.: Solid state fermentation with Cordyceps militaris SN-18 enhanced antioxidant capacity and DNA damage protective effect of oats (Avena sativa L.). - J. Funct. Foods 16: 58-73, 2015. 